\title{
TAHSP:-
}

The Internet Joưnal of Allied Health Sciences and Practice

A Peer Reviewed Publication of the College of Health Care Sciences at Nova Southeastern University

Dedicated to allied health professional practice and education

http://ijahsp.nova.edu Vol. 13 No. 1 ISSN 1540-580X

\section{Reliability of Breathing Rate Assessment and Chest Expansion Measurement: A Pilot Study in Typically Developing Children}

\author{
Mary-Grace De La Peña, PTRP 1 \\ Mary Grace Jordan, RPT, PTRP2 \\ Edward James Gorgon, MPhysio, PTRP3
}

1. Instructor, Department of Physical Therapy, University of the Philippines Manila, Manila, Philippines

2. Home Health Physical Therapist, Kare Partners/ALACARE Home Health and Hospice, Scottsboro, Alabama

3. Assistant Professor, Department of Physical Therapy, University of the Philippines Manila, Manila, Philippines

Philippines \& United States of America

CITATION: De La Peña M, Jordan M, Gorgon E. Reliability of Breathing Rate Assessment and Chest Expansion Measurement: A Pilot Study in Typically Developing Children. The Internet Journal of Allied Health Sciences and Practice. January 2015. Volume 13 Number 1.

\section{ABSTRACT}

Purpose: Despite the apparent acceptance of breathing rate assessment and chest expansion measurement (CEM) among physical therapists, little is known about the measurement properties of these tests and measures especially when administered to children. Reference data from typically developing children have been reported in the literature without investigating the reliability of measurements. This pilot study aimed to systematically explore the feasibility and inter-rater and test-retest reliability of these chest examination methods in children. Method: Nineteen children, 10 males and 9 females, with mean (SD) age of $11.11( \pm 1.29)$ years, were selected through convenience sampling. Assessors measured the breathing rate and chest expansion of the participants using a well-defined protocol. A second assessment was conducted after two weeks under similar testing conditions. Results: Breathing rate assessment yielded poor reliability across raters and testing occasions. CEM taken at the level of the fourth intercostal space revealed poor to excellent reliability (ICC $=0.48$ to 0.81 ), while measurements taken along the xiphoid process produced acceptable inter-rater reliability (ICC $=0.6$ to 0.7 ) and excellent test-retest reliability (ICC $=0.88$ to 0.94 ). Conclusions and Recommendations: CEM may be feasible and reliable to use on typically developing children given a welldefined protocol. Assessment of breathing rate may need to be evaluated further for clinical acceptability given potentially poor reliability in children.

\section{INTRODUCTION}

Pulmonary conditions are among the leading causes of morbidity and mortality in the pediatric population. In 2011, pneumonia and other acute respiratory infections were the causes for $13 \%$ of deaths in neonates and children under five years globally. ${ }^{1}$ In the Philippines, the latest available data from the Department of Health indicate that pneumonia is the third leading cause of child mortality in the pediatric age groups while acute lower respiratory tract infection, pneumonia, bronchitis, and respiratory tuberculosis rank among the top ten leading causes of morbidity. ${ }^{2,3}$ Individuals with primary or secondary pulmonary disorders are not the only ones facing the disease burden, as typically developing children are also at risk of acquiring pulmonary conditions due to their increasing exposure to pollution and passive smoking. ${ }^{4-6}$ Pulmonary disorders can result in limitations in movement and physical function due to the inadequate delivery of oxygen to skeletal muscles either during rest or physical activity. ${ }^{7}$ 
With its unique emphasis on movement as a primary means of promoting health and preventing disease and disability, physical therapy is well positioned to enhance function in children with impairment in pulmonary function. ${ }^{8}$ Physical therapists provide a range of therapeutic exercises and other interventions to address impairment and increase functional ability, and monitor the progress of intervention to optimize patient care. 9,10 Sound assessment of pulmonary function provides the necessary foundation for sound physical therapy intervention. Pulmonary assessment comprises history-taking, review of systems, and performance of tests and measures, and forms an important component of overall assessment in various physical therapist practice dimensions. ${ }^{9}$ Chest examination methods, such as chest expansion measurement (CEM) and quantifying breathing rate, are among the tests and measures conducted by physical therapists to determine the status of ventilation and respiration in patients. 9,11

Despite the apparent acceptance of chest examination methods among physical therapists, little is known about the measurement or psychometric properties of these methods especially when administered on children. Evaluation of psychometric properties typically starts with an exploration of the reliability and practicality of administration of tests and measures in the population for which these are intended to be used. ${ }^{12}$ Reliability is the repeatability and dependability of a measurement procedure or consistency of a measurement instrument in producing the same result.13,14 Practicality of administration includes requirements of the test related to administration time, experience and training of assessors, equipment, format, and method of scoring, and is often determined in a feasibility study prior to implementing a main study. ${ }^{15,16}$

To date, there is limited evidence on the reliability of chest examination methods in children. One study has reported estimates of intra-rater reliability $(I C C=0.95)$ and inter-rater reliability $(I C C=0.85)$ for $C E M$, but this study was limited to children and adolescents with cystic fibrosis (CF) in the age range of 5.9 to 18.9 years. ${ }^{17}$ Meanwhile, a study conducted among children aged 15 days to 3 years and with no diagnosed respiratory conditions demonstrated good reliability of breathing rate assessed using a stethoscope. ${ }^{18}$ Reference data have been reported for breathing rate and CEM for healthy children and children with cerebral palsy (CP) who were 4 years of age and older despite the lack of reliability studies to support the use of the measurement methods. 19,20 The insufficient evidence for the measurement properties of clinical chest examination methods despite widespread use underscores an important gap in the scientific literature that underpins pediatric physical therapist practice. This pilot study aimed to systematically explore the feasibility and inter-rater and test-retest reliability of breathing rate assessment and CEM in children with typical development (TD). Findings of this study can add to the available information on both the usefulness and limitations of chest examination procedures, as well as provide insights on improving test administration for use in both clinical and research practice.

\section{METHOD}

This pilot study utilized an observational, methodological design in investigating the properties of the measurement procedures. ${ }^{21}$ The study was approved by the ethics review committee of the University of the Philippines Manila - College of Allied Medical Professions.

\section{Participants}

Nineteen children with TD, 10 males and 9 females, with mean (SD) age of $11.11( \pm 1.29)$ years, constituted the sample. Specifically, 19 children participated in the inter-rater reliability testing while 8 children returned for test-retest reliability testing. Participants were sampled conveniently. The study protocol was explained to all participants and their parents prior to obtaining written consent from the participants' parents or legal guardians. The participants provided verbal assent before undergoing testing.

\section{Materials}

A printed module containing standardized steps and instructions was used by the assessors in carrying out the procedures. Digital stopwatches were employed in the assessment of breathing rate while standard cloth measuring tapes were utilized in quantifying chest wall expansion. A digital thermometer was used to ensure that none of the participants had fever at the time of testing. Assessment data were recorded on forms created specifically for the study.

\section{Test protocol}

Two of the authors (MGMJ and EJRG) conducted the examination procedures. Both assessors were licensed physical therapists with clinical experience in both pediatric and adult physical therapy. No additional training on administering the pulmonary assessment procedures was provided to the assessors. All assessments were made independently by the two assessors. The assessors administered the tests to the participants in adjacent rooms under similar conditions (similar room temperature, time of assessment, measurement materials, physical setup, and assessment procedures) based on the study protocol. A random order of participants to be tested was generated for each assessor. A second assessment was conducted after two weeks under similar testing conditions. Data from the first assessment were not made available to the assessors during the second

(c) The Internet Journal of Allied Health Sciences and Practice, 2015 
assessment to minimize bias.

Breathing rate was assessed unobtrusively (i.e. without informing the participant that breathing cycles were being counted). The participant was placed in a short-sitting position, with the assessor seated facing the participant at a 45-degree angle. Breathing rate was measured by counting the number of cycles of relaxed inhalation and exhalation in one minute. ${ }^{22,23}$ The assessor placed one hand over the chest of the participant for easier monitoring of cycle count.

Amount of chest wall expansion was quantified by determining the difference between chest circumference measurements during maximal voluntary inspiration and maximal voluntary expiration..$^{20}$ To measure maximal voluntary inspiration, participants were instructed to hold a deep, full breath for three seconds. To measure maximal voluntary expiration they were instructed to exhale fully. A standard cloth measuring tape was wrapped around the chest at the levels of the fourth intercostal space and xiphoid process that represented the body landmarks for upper chest and lower chest movements respectively. Three trials of CEM were conducted and scores were averaged and recorded. CEM was obtained with the participant in short-sitting position and with the assessor situated posterior to the participant.

\section{Data Analysis}

To determine inter-rater reliability and test-retest reliability estimates of the continuous variables, intraclass correlation coefficients (ICC) with $95 \%$ confidence intervals $(\mathrm{Cl})$ were calculated. Reliability estimates were interpreted as follows: $\geq 0.80=$ excellent, 0.60 to $0.79=$ acceptable reliability, and $<0.60=$ poor reliability. ${ }^{24}$

\section{RESULTS}

Inter-rater and test-retest reliability of breathing rate assessment and CEM

Reliability estimates for breathing rate assessment and CEM are detailed in Table 1. Breathing rate assessment yielded poor reliability estimates across assessors and testing occasions. Estimates for chest expansion measurements taken at the level of the fourth intercostal space were poor for inter-rater reliability and acceptable to excellent for test-retest reliability. Measurements obtained at the level of the xiphoid process produced consistently higher ICC values than those taken at the level of the fourth intercostal space, yielding acceptable inter-rater reliability and excellent test-retest reliability.

Table 1. Results of inter-rater and test-retest analyses of breathing rate assessment and CEM

\begin{tabular}{|c|c|c|c|c|c|c|c|c|}
\hline & \multicolumn{4}{|c|}{ Inter-Rater Reliability } & \multicolumn{4}{|c|}{ Test-Retest Reliability } \\
\hline $\begin{array}{l}\text { Assessment } \\
\text { Procedure }\end{array}$ & \multicolumn{2}{|c|}{ Test 1} & \multicolumn{2}{|c|}{ Test 2} & \multicolumn{2}{|c|}{ Assessor 1} & \multicolumn{2}{|c|}{ Assessor 2} \\
\hline & $\mathrm{ICC}$ & $95 \% \mathrm{Cl}$ & ICC & $95 \% \mathrm{Cl}$ & ICC & $95 \% \mathrm{Cl}$ & ICC & $95 \% \mathrm{Cl}$ \\
\hline Breathing rate & 0.41 & $(-0.44$ to 0.8$)$ & 0.34 & $(-0.96$ to 0.78$)$ & 0.06 & $(-3.27$ to 0.81$)$ & 0.45 & $\begin{array}{ll}(-1.49 & \text { to } \\
0.89) & \\
\end{array}$ \\
\hline CEM & & & & & & & & \\
\hline $4^{\text {th }}$ intercostal space & 0.48 & $(-0.17$ to 0.82$)$ & 0.52 & $(-0.34$ to 0.86$)$ & 0.7 & $(-0.37$ to 0.94$)$ & 0.81 & $\begin{array}{ll}(0.21 & \text { to } \\
0.96) & \end{array}$ \\
\hline Xiphoid process & 0.6 & $(-0.36$ to 0.89$)$ & 0.7 & $(-0.29$ to 0.92$)$ & 0.88 & (0.47 to 0.98$)$ & 0.94 & $\begin{array}{ll}(0.74 & \text { to } \\
0.99) & \end{array}$ \\
\hline
\end{tabular}

\section{DISCUSSION}

This pilot study sought to provide useful information regarding the reliability and practicality of breathing rate assessment and CEM in children. Breathing rate assessment and CEM are conventional assessment procedures utilized by physical therapists to clinically determine ventilatory function in children in clinical practice, yet there is limited evidence regarding the measurement properties and practical aspects of administration of these methods.

\section{Reliability of Breathing Assessment}

Findings demonstrate that breathing rate measurements may not be easily reproducible in children even when the assessment procedure has been well defined and testing conditions have been made very consistent. This can pose an important problem since measurements that cannot be reproduced are of limited value to clinicians. Only one other study has investigated reliability in children but this was carried out in children who were 15 days to 3 years of age and the results might not apply to older 
children. ${ }^{18}$ The poor reliability of breathing rate assessment in this study parallels the findings of an earlier study involving adult patients in an urban hospital which similarly utilized careful measurement of breathing rate. ${ }^{25}$ Participants' knowledge of the assessment technique has been shown to alter breathing rate. ${ }^{26}$ The participants in this study were not made aware that their breathing rate was being assessed and thus such factor did not likely influence the participants' breathing. Breathing rate can also be affected directly by an increase in body temperature or physical activity. ${ }^{27,28}$ Given children's propensity to engage in active play whether on their own or with each other, efforts were made to control the participants' level of physical activity prior to testing by asking them to rest for several minutes before commencing the assessments.

The poor reliability of breathing rate assessment has important negative ramifications on the validity of assessment results. Despite this, clinical methods of breathing assessment are still widely used by physical therapists to detect tachypnea or bradypnea and to guide decision making in exercise prescription. ${ }^{11}$ There is a need, therefore, to rationalize the value of breathing rate assessment in pediatric clinical assessment. Further, an important question may be raised regarding the usefulness of published reference data for breathing rate derived from children with TD. 18,19 Certain strategies may improve reliability such as dichotomizing the classification of breathing rate into bradypnea (abnormally slow breathing) and tachypnea, defined as greater than 27 breaths/minute, which has been shown to result in moderate reliability $(k=0.6) .{ }^{25}$ Ultimately, however, results of breathing rate assessment should not be interpreted in isolation and should be used with careful consideration of other assessment findings in ascertaining pulmonary status in clients..$^{25}$

\section{Reliability of CEM}

CEM taken at the level of the fourth intercostal space revealed varied reliability estimates whereas measurements taken along the xiphoid process produced consistently higher reliability estimates for both inter-rater reliability and test-retest reliability. This is likely related to the xiphoid process being a more stable landmark and therefore more easily and accurately palpated. ${ }^{29}$ The finding was consistent with the high intra-rater and inter-rater reliability estimates for CEM obtained in studies that employed similar methods on children and adolescents with CF and healthy adult males. ${ }^{17,30}$ It would be reasonable to hypothesize that CEM may be taken effectively at the level of the xiphoid process in routine clinical assessment.

\section{Feasibility}

Specific features of clinical assessments are known to contribute to safety and practicality in administration. ${ }^{15}$ Many of such features were covered in the pulmonary assessment protocol used in this study. The protocol specified the testing conditions, needed materials, and procedures to be performed for each area of assessment, including participant position, therapist position, and verbal instructions. The protocol also provided a definition of breathing rate assessment and description of the landmarks for CEM to guide the assessors in measuring and recording results. Use of a well-defined protocol was particularly helpful in the absence of any highly specialized training or experience in pulmonary assessment between the two assessors. In total, assessment of each participant required only 10 minutes or less. Assessment employed materials that were not costly and were often available in conventional clinical settings. All these lend support to the practicality of administration of breathing rate assessment and CEM on children with TD. Conducting the tests on children might benefit from creative means of promoting relaxation prior to administration given children's natural tendency to engage in active play.

\section{Limitations and Recommendations}

This pilot study was limited to children who were typically developing and thus the results might have some generalizability in typically developing children who have acquired a pulmonary disorder. The results may not necessarily be generalized to other sub-groups in the pediatric population such as those with neuromotor conditions and cognitive impairment. As a pilot study, however, the main purpose was not to establish definitive results but rather to explore the feasibility of methods and gather preliminary data for generating hypotheses. ${ }^{31}$ The use of a standardized methodology in this study allowed for preliminary positive findings to be generated for CEM within the limits of a pilot study design, suggesting that such methodology might be useful in future studies of wider scope. A logical next step would be to explore the measurement properties of CEM in children with either primary or secondary pulmonary conditions. Studies that employ a more rigorous design, including a sufficiently large sample size, and that involve patients with diagnosed health conditions other than CF are recommended to achieve maximum variation and contribute to clearly defining the level of reliability of the assessment procedures. ${ }^{32}$ How these manual assessment procedures relate to conventional laboratory-based measurements of pulmonary function can also be explored.

\section{CONCLUSION}

This pilot study has shown that CEM may be feasible and reliable to use on children given a well-defined protocol. Breathing rate assessment appears to be of poor reliability and may warrant justification for routine use in clinical practice. It is hoped that this pilot study will provide the impetus for additional research on the measurement properties of clinical pulmonary assessment methods for children, specifically those with impaired health. 


\section{ACKNOWLEDGEMENTS}

The authors thank Rolabel Ma. Lin Cuevas, Sheryll Anne Manalili, Adela Grace Patungan and Exiquiel Pimentel for their invaluable contribution in participant recruitment and data collection. Permission to be acknowledged in the study was obtained from the individuals.

\section{REFERENCES}

1. World Health Organization [Internet]. Children: reducing mortality. [cited 2012 Jan 5]. Available from: http://www.who.int/mediacentre/factsheets/fs178/en/index.html

2. Department of Health [Internet]. Top (10) Leading Causes of Child Mortality in the Philippines. [cited 2012 May 17].Available from: http://www.doh.gov.ph/kp/statistics/child_mortality

3. Department of Health [Internet]. Leading Causes of Morbidity. [cited 2012 May 22]. Available from: http://www.doh.gov.ph/kp/statistics/morbidity

4. Venners SA, Wang X, Changzhong C, Wang B, Ni J, Jin Y. Exposure-response relationship between paternal smoking and children's pulmonary function. Am J Respir Crit Care Med. 2001;164(6):973-6. [PMID 11587981]

5. Garcidueñas LC, Tiscareño AM, Fordham LA, Chung CJ, Salazar GV, Gómez SF. Lung radiology and pulmonary function of children chronically exposed to air pollution. Environ Health Perspect. 2006;114(9):1432-7. [PMID 16966101]

6. Frye C, Hoelscher B, Cyrys J, Wjst M, Wichmann HE, Heinrich J. Association of lung function with declining ambient air pollution. Environ Health Perspect. 2003;111(3):383-7. [PMID 12611668]

7. Peel C. The cardiopulmonary system and movement dysfunction. Phys Ther. 1996;76:448-55. [PMID 8637934]

8. World Confederation for Physical Therapy [Internet]. Policy statement: Description of physical therapy. [cited 2012 May 22]. Available from: http://www.wcpt.org/policy/ps-descriptionPT

9. American Physical Therapy Association. Guide to Physical Therapist Practice, $2^{\text {nd }}$ ed. Phys Ther. 2001; 81(1): 9-746. [PMID 11175682]

10. Kisner C, Colby LA. Therapeutic Exercise. 6th ed. Philadelphia: F.A. Davis Company; 2012.

11. Tecklin JS. Pediatric physical therapy. 5th ed. Philadelphia: J.B. Lippincott Company; 2014.

12. Switzer GE, Wisniewski SR, Belle SH, Dew MA, Schultz R. Selecting, developing, and evaluating research instruments. Soc Psychiatry Psychiatr Epidemiol. 1999;34(8):399-409. [PMID 10501709]

13. Cicchetti DV, Rourke BP. Methodological and Biostatistical Foundations of Clinical Neuropsychology and Medical and Health Disciplines. 2nd ed. London: Taylor \& Francis Group; 2004.

14. Di lorio CK. Measurement in Health Behavior: Methods for Research and Education. San Francisco: Jossey-Bass; 2005.

15. VanSwearingen JM, Brach JS. Making geriatric assessment work: selecting useful measures. Phys Ther. 2001;81(6):123352. [PMID 11380279]

16. Arain $\mathrm{M}$, Campbell $\mathrm{MJ}$, Cooper $\mathrm{CL}$, Lancaster $\mathrm{GA}$. What is a pilot or feasibility study? A review of current practice and editorial policy. BMC Med Res Methodol. 2010;10:67-73. [PMID 20637084]

17. Custer JWH, Arets HGM, Engelbert RHH, Kooijmans FTC, van der Ent CK, Helders PJM. Thoracic excursion measurement in children with cystic fibrosis. J Cyst Fibros. 2005;4(2):129-33. [PMID 15914097]

18. Rusconi F, Castagneto M, Gagliardi L, Leo G, Pellegatta A, Porta N, Razon S, Braga M. Reference values for respiratory rate In the first 3 years of life. Pediatrics. 1994;94(3):350-5. [PMID: 8065862]

19. Wallis LA, Healy M, Undy MB, Maconochie I. Age related reference ranges for respiration rate and heart rate from 4 to 16 years. Arch of Dis Child. 2005;90(11):117-21. [PMID 16049061]

20. Ersöz M, Selçuk B, Gündüz R, Kurtaran A, Akyüz M. Decreased chest mobility in children with spastic cerebral palsy. Turk J Pediatr. 2006;48(4):344-50. [PMID 17290570]

21. Portney L, Watkins M. Foundations of Clinical Research: Applications to Practice. 3rd ed. New Jersey: Pearson Prentice Hall; 2009.

22. Kelsey J, McEwing G. Clinical Skills in Child Health Practices. Edinburgh: Elsevier; 2008.

23. Ragnarsdóttir MR, Kristinsdóttir EK. Breathing movements and breathing patterns among healthy men and women 20-69 years of age. Respiration. 2006;73(1):48-54. [PMID 16106113]

24. Law M. Outcome measures rating form. CanChild Centre for Disability Research, Ontario; 2004. Available from http://www.canchild.ca/en/canchildresources/resources/measguid.pdf

25. Edmonds ZV, Mower WR, Lovato LM, Lomeli R. The reliability of vital signs measurement. Ann Emerg Med. 2002;39:233-7. [PMID 11867974]

26. Gilbert R, Auchincloss JH, Brodsky J, Boden W. Changes In Tidal Volume, Frequency, and Ventilation Induced By Their Measurement. J Appl Physiol. 1972;33(2):252-4. [PMID 5054434]

27. Guyton AC. Human Physiology and Mechanism of Disease. 5th ed. Philadelphia: W.B. Saunders Company; 1992.

28. Kispert CP. Clinical Measurements to Assess Cardiopulmonary Function. Phys Ther. 1987;67:1886-90. [PMID 3685117] 
29. Leaver D, Washington C. Principles and Practice of Radiation Therapy: Physics, Simulation, and Treatment Planning. Michigan: Mosby; 1996.

30. Bockenhauer SE, Chen H, Julliard KN, Weedon J. Measuring Thoracic Excursion: Reliability of the Cloth Tape Measure Technique. J Am Osteopath Assoc. 2007;107(5):191-6. [PMID 17596587]

31. Lancaster GA, Dodd S, Williamson PR. Design and Analysis of Pilot Studies: Recommendations for Good Practice. J Eval Clin Pract. 2002;10(2):307-12. [PMID: 15189396]

32. Brooks D, Thomas J. Interrater Reliability of Auscultation of Breath Sounds Among Physical Therapists. Phys Ther. 1995;75(12):1082-8. [PMID 7501711] 